be improved not only by a judicious use of a ruler to draw straight lines but by the addition of legends to supplement references in the text.

The book is, in my view, a brilliant essay for the plain man, particularly tor, who wants to understand how experts study complexity. It is a generous bequest from one of the most distinguished biologists of his generation. It deserves to be re-issued for a for the plain politician and administra-

wider public as a paperback at a moderate price; but it would be a courtesy to the memory of Hal Waddington to re-edit the book conscientiously before it is re-issued.

Lord A shby was formerly Master of Clare College, Cambridge. He is Chancellor of the Queen's University, Belfast, and is at present Walgreen Professor of Human Understanding at the University of Michigan.

\section{Mathematical treasure}

Proofs and Refutations: The Logic of Mathematical Discovery. By Imre Lakatos. Edited by John Worrall and Elie Zahar. Pp. xii +174 . (Cambridge University: Cambridge, London and New York, 1976.) Hardcover £7.50; paperback $£ 1.95$.

For anyone interested in mathematics who has not encountered the work of the late Imre Lakatos before, this book is a treasure; and those who know well the famous dialogue, first published in 1963-64 in the British Journal for the Philosophy of Science, that forms the greater part of the book, will be eager to read the supplementary material. The dialogue is what Lakatos calls a "rational reconstruction of history", that is, the history of the Euler conjecture on polyhedra $(V-E+F=2)$; the purpose is to illustrate principles of methodology. The participants seem themselves to be more interested in methodological questions than in the mathematical problem, which imparts a somewhat ponderous tone to the discussion; but the reconstruction, and the actual historical information given in the extensive footnotes, are in themselves fascinating. The supplementary matter, extracted by the editors of this posthumous work from earlier unpublished writings by Lakatos, and not revised by him before his death, consists, first, of a conclusion to the dialogue, in the form of a discussion of Poincaré's proof, and, secondly, of much briefer case studies, not in dialogue form, of examples from analysis and measure theory.

The original dialogue, without this supplementary material, was stimulating but tantalising, in that it was unintended his readers to draw. Plainly, clear just what conclusions Lakatos one is a methodological thesis, concerning the process of mathematical discovery. According to Lakatos, the best model for this process is not the linear, Euclidean one of indubitable deductions from an initial set of axioms and definitions, but that of an interplay between ideas for proofs and counterexamples in the light of which the proof is progressively improved. We begin, typically, with a conjecture and a tentative proof. We should then seek for counter-examples, guided by the proof: when these are found, we should not rule them out by ad hoc ("monster-barring") definitions, even when it can plausibly be claimed that our original concepts, as we vaguely apprehended them, did not cover those examples; nor should we merely make an ("exception-barring") restriction of the domain of application of the theorem so as to exclude the counterexamples. Rather, the proof must be re-analysed in the light of the counterexamples: in this way, we discover hidden lemmas, and build into the statement of the theorem the hypotheses essential for its validity.

With these maxims about how to do mathematics, few would disagree, despite a certain truculence in Lakatos's manner of arguing for them. He has, however, another thesis, about how mathematics should be presented. $\mathrm{He}$ deplores the deductivist Euclidean style, in which a set of complicated definitions and axioms are stated at the outset, without explanation, and complex theorems follow, with their proofs in the barest form. Exposition in this manner conceals what is required for understanding: instead, it should reflect the actual history of the problems, "rationally reconstructed", so that the motivation of the definitions, axioms and hypotheses of the theorems becomes apparent. This second thesis, about mathematical style, is more tendentious than the first, but has a great deal to be said in its favour. Certainly it impedes understanding when a definition is given without any illustration of the cases to which the term so defined does and does not apply, and without any explanation why it should be framed as it is; and certainly also the historical information embedded in most mathematical textbooks is often mythological. How much difference would be made to the best mathematical expositions by the adoption of
Lakatos's recommendations depends on how much is allowable as "rational reconstruction".

The most important question, however, is how far Lakatos intended to assert, as well as a methodological and a stylistic thesis, a philosophical one. Most people's first reaction to Lakatos's work is to say that the situation he describes is one likely to prevail in a branch of mathematics in its early stages, before it has attained the condition of an axiomatised theory, but not when it has reached maturity. On this view, Lakatos's observations have no philosophical consequences: they describe how we do, or can best, arrive at a developed theory, how we can make mathematical discoveries, not what such a theory is like when we have succeeded in framing it. There are, however, passages (pages 100 and 138) suggesting that Lakatos took a quite different view, namely that the process of finding counter-examples and re-analysing proofs in the light of them is never-ending, or, at least, that we can never know that we have reached an end.

This thesis, if accepted, would indeed be of critical philosophical importance, since it would demand, though it would not itself provide, a revision of most of the known accounts of mathematical knowledge, if not of mathematical truth. Other passages (for example, p. 124), however, suggest that the process does terminate. The editors are more clearly of this opinion than Lakatos himself (pages 125-6 and 138n). They think that, by formalisation, one can dispel all doubt from the proof, and throw it upon the axioms: from these, however, it can never be removed. This leaves a big lacuna in the whole discussion: the character of doubt concerning the axioms of a mathematical theory is far from obvious. Thus, in the upshot, we are presented, in this book, with a piece of work which, although admirable as a historical study, is, from a philosophical standpoint, incomplete and inconclusive. Doubtless the delay in publication was due to Lakatos's awareness of this: had he lived, we should presumably have eventually had a more finished work.

Nevertheless, the book, as it stands, is rich and stimulating, and, unlike most writings on the philosophy of mathematics, succeeds in making excellent use of detailed observations about mathematics as it is actually practised.

Michael Dummett

Michael Dummett was Reader in the Philosophy of Mathematics at the University of Oxford from 1961-74, and has been Senior Research Fellow at All Souls' College, Oxford, UK, from 1974. 